\title{
Apparent depth of pictures reflected by a mirror: The plastic effect
}

\author{
Atsuki Higashiyama $\cdot$ Koichi Shimono
}

Published online: 13 July 2012

(C) Psychonomic Society, Inc. 2012

\begin{abstract}
We investigated the plastic effect in picture perception, in which the apparent depth of a picture is increased when it is reflected by a mirror. The plastic effect was well known in the mid-18th century, but very few studies have elucidated its nature. In Experiment 1, we examined how often the plastic effect occurs in different ocular conditions. A group of 22 observers compared directly observed pictures and their mirror-reflected images in each of freebinocular, free-monocular, and restrictive-monocular conditions. When the observers were forced to choose the picture that appeared greater in depth, $73 \%$ of them chose the reflected pictures, regardless of oculomotor condition. In Experiment 2, we examined how often the plastic effect is detected as a function of observation time. When 22 observers compared a directly watched movie and its mirrorreflected movie for $5 \mathrm{~min}$, the number of observers who judged the reflected movie to be greater in depth was about $55 \%$ at the onset of the trial but was $86 \%$ at the end. In Experiment 3, we examined transfer of the plastic effect. Ten observers judged the change in apparent depth of directly observed pictures after prolonged exposure to the same reflected or actual pictures. Transfer was confirmed and was greater for pictures that represented greater depth $(r=.88)$. We suggested that the plastic effect is mainly induced by the double apparent locations of a reflected picture. From the long incubation time and the transfer to real pictures, we also suggested that it involves perceptual learning regarding visual skill.
\end{abstract}

\footnotetext{
A. Higashiyama $(\bowtie)$

Department of Psychology, Ritsumeikan University,

Tojiin-kitamachi, Kita-ku,

Kyoto, Japan

e-mail: achan@1t.ritsumei.ac.jp

K. Shimono

Tokyo University of Marine Science and Technology,

Tokyo, Japan

Keywords 3-D perception - Scene perception · Spatial localization

In this article, we investigate the plastic effect: If a single flat picture is seen in a mirror, through a convex lens, or by means of the combination of mirror and lens, the depth of the picture appears greater than the depth of the directly observed picture (Schlosberg, 1941). This effect has been vividly described: "In a typical snapshot of a person against a mixed background, the person stands out clearly, and plastic space can be seen between him and the background, as well as between the objects of the near and far background" (Schlosberg, 1941). The plastic effect can be emphasized by using other ingenious optic devices-for instance, a zograscope (Chaldecott, 1953), a synopter (Carl Zeiss, 1907, introduced by Koenderink, van Doorn, \& Kappers, 1994), a plastoscope (Zoth, 1916), Javal's iconoscope (Tscherning, 1904), and even a stereoscope in which the same picture is presented to each eye (Emerson, 1863).

The equipment and specific pictures by which the plastic effect is produced were popular among wealthy people in Europe and Japan in the mid-18th century (Clayton, 1997; Oka, 1992; Screech, 1996). For example, Fig. 1 indicates that a young girl of that time enjoyed watching a picture with a zograscope, which was probably conveyed from Europe. Scientific studies appeared early in the last century, when interest in stereoscopy was high (Ames, 1925; Claparède, 1904; Eaton, 1919; Ponzo, 1911; von Kalpinska, 1910; Zoth, 1916), but no study has clearly explained why apparent depth is increased in the reflected picture. In our study, we did address this question, and for simplicity, we used pictures that were reflected by mirror(s).

Although the plastic effect itself has not been explained clearly, it has been observed that oculomotor conditions may affect the plastic effect. Ames (1925) and Claparède (1904), comparing the apparent depths of a picture between binocular and monocular viewings, reported that the plastic effect 


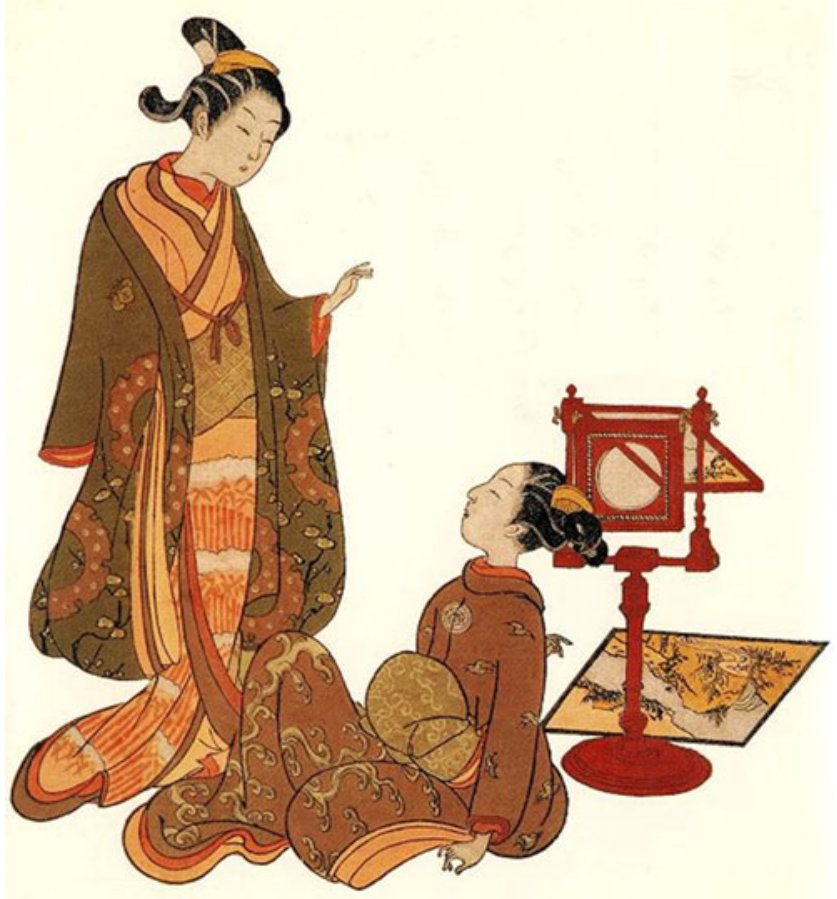

Fig. 1 A young girl looking into a zograscope. The picture on the floor is reflected by a mirror and passes through a convex lens to reach the eyes. The illustration is a modified version of Koya no Tamagawa by Harunobu Suzuki (1725?-1770), which was taken from Oka's (1992) book cover

was apt to occur for monocular rather than for binocular viewing. Interestingly, this observation is in contrast with the apparent depth between objects in a three-dimensional scene, which is reduced for monocular rather than binocular viewing (Allison, Gillam, \& Vecellio, 2009; Foley, 1980; Frisby, Buckley, \& Duke, 1996; Gogel, 1962; Grant, 1942; Koenderink, van Doorn, \& Kappers, 1995; Komoda \& Ono, 1974; McKee \& Taylor, 2010; Swenson, 1932).

Schlosberg (1941) explained augmentation of the plastic effect in monocular conditions by the reduction of "flatness" cues. According to this explanation, when we see a picture directly with both eyes, we see it either as a picture representing depth or as actual objects deployed in depth. The picture may contain both flatness cues (i.e., binocular retinal disparity, binocular convergence, surface glare, cues from accommodation, failure to obtain change in motion parallax when the picture or the observer is moved, and frame of the picture) and pictorial depth cues (i.e., shading, clearness of outlines, perspective, and superposition). In monocular viewing, binocular retinal disparity and binocular convergence are removed from the available flatness cues, so that pictorial depth cues are relatively effective and may increase the apparent depth of the picture. Although Schlosberg speculated that a mirror also breaks up flatness cues, it was not clear how it would do so.

Development of the technique of computer display has made it possible to measure the apparent slant and depth of an object represented in a picture (Bülthoff \& Mallot, 1988; Stevens \& Brooks, 1987), and Koenderink, van Doorn, and Kappers $(1992,1994,1995)$ were probably the first to measure the effects of oculomotor cues on the plastic effect. In particular, Koenderink et al. (1994) obtained local surface attitudes by adjusting a gauge figure so as to fit the apparent surfaces of an object represented under binocular viewing through a synopter (i.e., a mirror device), under natural monocular viewing, and under natural binocular viewing. When a relief of the object was recovered from the local surface attitudes, an individual difference was found. For one observer, the depth was greatest for binocular viewing through a synopter, was modest for natural monocular viewing, and was least for natural binocular viewing. For two other observers, the depths for synoptic binocular and natural monocular viewing did not differ, but both were greater than the depth from natural binocular viewing. It was thus difficult to draw a unique conclusion about the effects of a mirror on depth perception.

In the process of reviewing the plastic-effect studies, we felt that the roles of a mirror and of depth or distance cues are unclear. To understand these roles, we first assumed that a mirror that reflects a picture localizes it at two places. This assumption can be understood by referring to Fig. 2, which illustrates the situation in which an observer compares a directly observed picture and the mirror-reflected picture, both of which are exposed only to the left eye, with the right eye occluded. In this condition, the directly observed picture is solely localized where it is placed, whereas the reflected picture is possibly localized where the mirror is placed and where the virtual image is formed. We also assumed that the distance from the observer to the reflected picture is unsettled by the double locations of that picture, so that instead

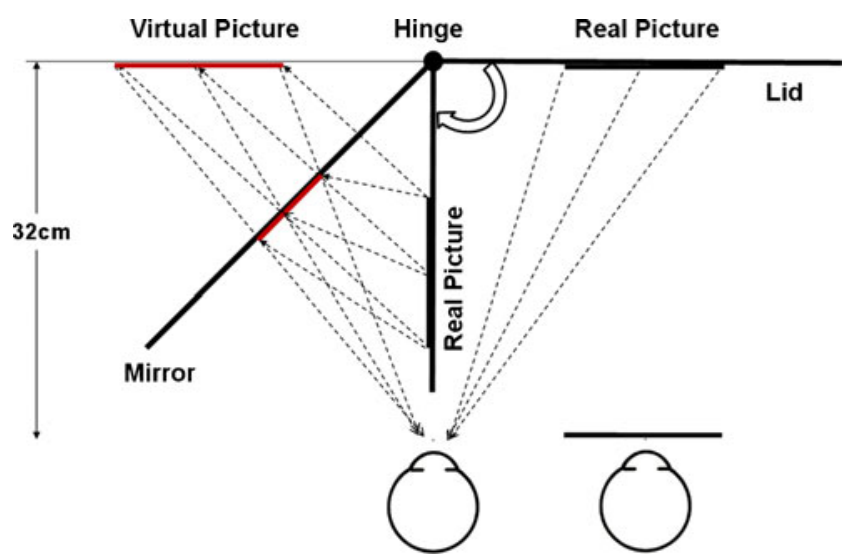

Fig. 2 The optical relations among a real picture, the reflected picture, a mirror, and the observer's left eye under restrictive monocular viewing in Experiment 1. When the observer looked at a real picture, it was placed in the frontoparallel plane, whereas when the observer looked at the reflected picture, the real picture was placed in the median plane and was reflected by a mirror that was slanted $45^{\circ}$ to the frontoparallel plane 
the effects of pictorial depth cues in the reflected picture are emphasized. In normal picture perception, flatness cues (i.e., a special state of distance cues) are potentially in conflict with depth cues (Gibson, 1979; Haber, 1980; Niederée \& Heyer, 2003; Pirenne, 1970; Rock, 1984). If the visual system faces such a cue conflict, it may tend to take the cues, for processing, that provide reliable or powerful information. We suppose that for a mirror-reflected picture, distance cues are less reliable, and depth cues are relatively enhanced in effectiveness.

With this idea in mind, in Experiment 1, we asked whether the plastic effect occurs at all for a picture reflected by a single mirror. We also asked how steadily the plastic effect is detected across different oculomotor conditions. Specifically, does the effect not occur in situations with rich flatness cues? Is it really augmented in situations with reduced distance cues? In the experiment, a large number of observers judged whether the apparent depth of reflected pictures was large or small, as compared to that of directly observed pictures, in conditions of free binocular, free monocular, or restrictive monocular viewing. The size of the plastic effect was indicated by the number of observers who judged the reflected pictures to be greater in depth.

If a mirror makes the absolute distance of reflected pictures unsettled because of their double locations, the plastic effect would be equally well detected across all oculomotor conditions. If, however, the reduction of flatness cues enhances the plastic effect, the effect would be reduced for free binocular viewing, during which the observers may be aware of the flat nature of the picture. The doublelocalization hypothesis and the reduced-flatness-cue hypothesis are not mutually exclusive; it is possible that the plastic effect would be detected in all oculomotor conditions, but that the strongest effect would be obtained in the reduced-oculomotor condition.

In Experiment 1, we also examined the relation between the plastic effect and the response time that is needed to judge depth. We assumed that if the plastic effect is increased, it would be easy to discriminate depth between directly observed pictures and the reflected pictures. This implies that a negative correlation could exist between the plastic effect and response time.

In addition to the double locations of a reflected picture and the reduction of flatness cues, observers' viewing attitude or "set" may be critical to the plastic effect. Schlosberg (1941) suggested that when we see a picture, the mode of perception - flatness or depth-is contingent on set. To understand the role of set in picture perception, consider, for example, that we see a picture in which a large, a medium, and a small circle are drawn at the left, center, and right positions in the picture, respectively. If we attend to the differences in visual angle among the circles, they are perceived as different-sized circles drawn in a picture. This is the flatness mode of perception, which is induced by a set for flatness. If we attend to the difference in depth among the circles, they are perceived as being the same size but at different depths (i.e., a relative-size cue to depth). This is the depth mode of perception, which is induced by a set for depth. A particular set may be formed by an observer's assumptions or may be induced by depth cues present in the visual field (Higashiyama, 1984).

To our knowledge, no one has explored how the formation of a depth set is related to the temporal properties of the plastic effect. We assume that for a depth set to be formed, a certain time is needed, and once the set has been formed, it lasts for a certain period of time. On the basis of this assumption, in Experiment 2 we examined how the plastic effect emerges over time: Observers were exposed to a real movie and to a reflected counterpart and, in the interim, repeatedly judged the movie that appeared to be greater in depth. As in Experiment 1, the size of the plastic effect was indicated by the number of observers who judged the reflected picture to be greater in depth. If the plastic effect were a simple process like the detection of light and sound, it would occur immediately after presentation of the reflected movie. If the plastic effect requires the formation of a depth set, it would be delayed.

In Experiment 3, we examined the sustainability of the plastic effect. If the effect is induced by formation of a depth set, it may persist for a while after viewing of the reflected pictures is terminated. To verify this prediction, we required observers to judge a change in the apparent depth of directly observed pictures after prolonged exposure to the reflected pictures. We assumed that the observers who experienced the plastic effect would learn to make use of pictorial depth cues and would ignore absolute distance cues. We also assumed that this acquired perceptual skill might be applied to directly observed pictures.

\section{Experiment 1}

\section{Method}

Observers A group of 66 students (23 men, 43 women; mean age 21.7 years) voluntarily participated in this experiment. They did not have any prior knowledge about the purpose of the experiment.

Apparatus A commercially available rear-surface silvered hand-mirror $(12 \mathrm{~cm}$ high $\times 15 \mathrm{~cm}$ wide $\times 0.2 \mathrm{~cm}$ deep $)$ was used that had a cover attached by a hinge (as is shown in Fig. 2). A picture $(8.8 \mathrm{~cm}$ high $\times 12.5 \mathrm{~cm}$ wide) was placed on the underside of the cover and was reflected by the mirror. When the observer faced toward the apparatus, he or she was able to see both the real picture and the 
reflected picture. The reflected picture was the left-right reversal of the real picture.

Stimuli and conditions Eleven pictures were used that were either collected from copyright-free sites on the Internet or taken by the authors: (a) night scene of downtown, (b) sculpture of a samurai, (c) train, (d) flower hedge, (e) woman in a hall, (f) girl in front of a wall, (g) woman picking up a button, (h) woman making a fist, (i) temple in Thailand, (j) restaurant, and (k) slope in a street. Figure 3 shows two representative pictures, (i) and (d), that greatly differed in depth cues.

The observers experienced three oculomotor conditions. In free binocular viewing, the positions of the real picture and the mirror were perfectly under the observer's control. Each observer held the mirror with one hand and the picture with the other hand and was able to change the angle between the mirror and the picture. Depending on the angle, the observers saw the real and reflected pictures simultaneously or successively. Since the operation of the angle was entirely left with the observers, some wanted to keep simultaneous views, and others preferred to keep successive views, but many observers alternated the simultaneous and successive views. The viewing distance of the pictures varied from 20 to $40 \mathrm{~cm}$, depending on different observers and different times. The observation was binocular without a bite-board. In free monocular viewing, all aspects of observation were the same as for free binocular viewing, except that one eye was covered. In restrictive monocular viewing, a real picture was presented in the frontoparallel plane at a viewing distance of $32 \mathrm{~cm}$, whereas the reflected picture was presented by reflecting the real picture in the median plane with a mirror that was slanted at $45^{\circ}$ to the frontoparallel plane (see Fig. 2). The cover, on the underside of which the real picture was placed, could pivot at the hinge and was alternatively placed in the frontoparallel and median planes. Thus, in this condition, the observers saw the real and reflected pictures successively. The mirror was fixed at the horizontal eye level.

Procedure To carry out a double-blind experiment, we employed three experimenters, one for each oculomotor condition. They were rigorously instructed as to how to run the experiment, but they were not informed of the outcome that was expected by the authors.

Twenty-two of the observers were randomly assigned to each of the oculomotor conditions. In the free binocular and monocular viewings, each observer held the apparatus with both hands and could change the angle between the mirror and the picture. The observer's face position was not fixed in either experiment. In the restrictive monocular viewing, the mirror was fixed, on a wooden base, at a slant of $45^{\circ}$ to the frontoparallel plane, and the picture was rotated by the experimenter so as to be in either the frontoparallel or the median plane, as required by the observer. The observer's face position was fixed with a head-and-chin rest.

In all conditions, the 11 pictures were randomly presented, one at a time, to each observer. On a given trial, each observer compared a directly observed picture and the reflected picture in a well-lit room. Half of the observers in each condition consistently reported the picture that appeared greater in depth, and the other half constantly reported the picture that appeared shallower in depth. The observers were instructed to base their judgments on their whole depth impression of each picture, not on the apparent depth between two specific points. The left-right positions of the mirror and the picture were counterbalanced among observers. No time limit was imposed on the observers in judging apparent depth.

In the binocular viewing, in addition to judgments of depth, the elapsed time from the presentation of a picture to the judgment of depth was measured in seconds. On a given trial, the experimenter put a picture on the open cover, started a stopwatch, and encouraged the observer to report the picture that appeared to be greater (or shallower) in depth. Once the observer had made the judgment, the experimenter stopped the stopwatch, noted the depth judgment and the response time, and exchanged the picture for the picture for the next trial. The time from when the stopwatch was stopped until the pictures were exchanged was about $10 \mathrm{~s}$. In the monocular viewing conditions, response time was not measured.
Fig. 3 Representative pictures used in Experiment 1: A temple in Thailand and a flower hedge. The left picture contains many depth cues (linear perspective, relative size, texture gradient, familiar size, interposition, light and shade, and implicit horizontal line), whereas the right contains fewer depth cues (interposition and light and shade)
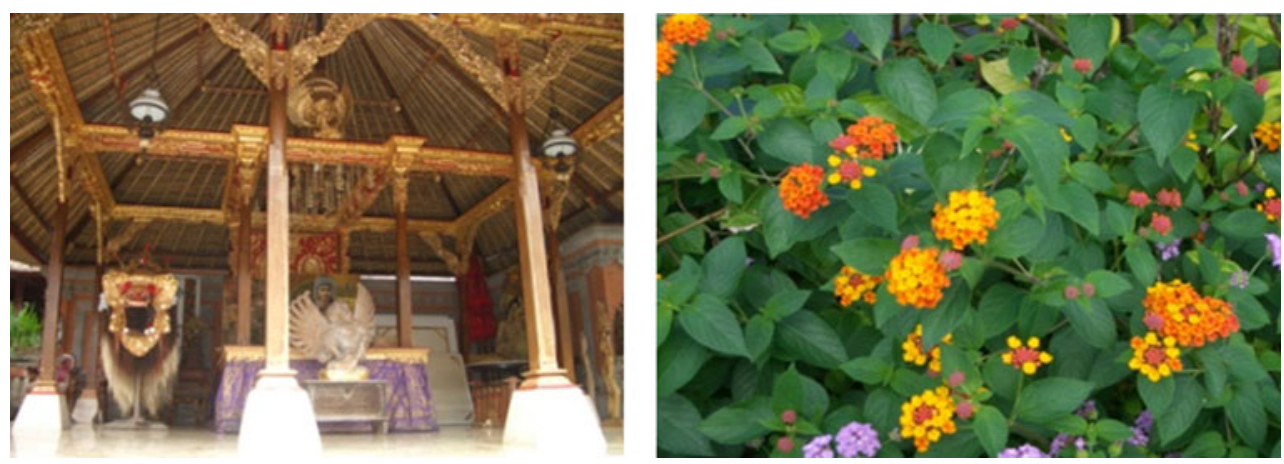


\section{Results}

Table 1 indicates the numbers of observers who judged each reflected picture to be greater in depth than the directly observed picture. If 16 or more observers judged this to be so, it meant that the reflected picture was judged to be significantly greater in depth (according to a binominal test, $p<.05$ ). The asterisks in Table 1 indicate such cases. For the freebinocular viewing condition, nine of the 11 pictures met this criterion; for the free-monocular condition, six pictures fell into this group; and for the restrictive-monocular condition, 10 pictures did so. Thus, in 25 of a total of 33 cases, the reflected pictures were judged to be greater in depth. In no case was a directly observed picture judged to be significantly greater in depth.

For each oculomotor condition, the mean number of reflections that each observer judged to be greater in depth significantly differed from chance (5.5): for free binocular viewing, $t(21)=5.34, p<.001$; for free monocular viewing, $t(21)=$ $6.32, p<.001$; for restrictive monocular viewing, $t(21)=6.99$, $p<.001$. For each oculomotor condition, the mean number of observers who judged each picture to be greater in depth significantly differed from chance (11): for free binocular, $t(10)=3.73, p<.001$; for free monocular, $t(10)=5.92$, $p<.001$; for restrictive monocular, $t(10)=14.64, p<.001$.

The presence or absence of the plastic effect depended on the picture, as is shown in Table 1. For six pictures (a, b, e, $h, i$, and $j$ ), the plastic effect was found for all conditions; for three pictures (c, f, and k), it was found in two conditions; for one picture $(\mathrm{d})$, it was found in one condition; for one

Table 1 Numbers of observers who judged each reflected picture to be greater in depth than the directly observed picture under free-binocular, free-monocular, and restrictive-monocular conditions

\begin{tabular}{lllll}
\hline Pictures & $\begin{array}{l}\text { Free } \\
\text { Binocular }\end{array}$ & $\begin{array}{l}\text { Free } \\
\text { Monocular }\end{array}$ & $\begin{array}{l}\text { Restrictive } \\
\text { Monocular }\end{array}$ & Mean (\%) \\
\hline (a) & $17^{*}$ & $16^{*}$ & $19^{*}$ & $17.3(79)$ \\
(b) & $18^{*}$ & $20^{*}$ & $17^{*}$ & $18.3(83)$ \\
(c) & $16^{*}$ & 14 & $18^{*}$ & $16.0(73)$ \\
(d) & 6 & 12 & $19^{*}$ & $12.3(56)$ \\
(e) & $16^{*}$ & $18^{*}$ & $16^{*}$ & $16.7(76)$ \\
(f) & $17^{*}$ & 12 & $16^{*}$ & $15.0(68)$ \\
(g) & 10 & 15 & 15 & $13.3(61)$ \\
(h) & $17^{*}$ & $17^{*}$ & $19^{*}$ & $17.7(80)$ \\
(i) & $20^{*}$ & $19^{*}$ & $16^{*}$ & $18.3(83)$ \\
(j) & $17^{*}$ & $16^{*}$ & $17^{*}$ & $16.7(76)$ \\
(k) & $16^{*}$ & 14 & $18^{*}$ & $16.0(73)$ \\
Mean (\%) & $15.5(70)$ & $15.7(71)$ & $17.3(79)$ & $16.1(73)$ \\
\hline
\end{tabular}

(a) Night scene of downtown; (b) Sculpture of a samurai; (c) Train; (d) Flower hedge; (e) Woman in a hall; (f) Girl in front of a wall; (g) Woman picking up a button; (h) Woman making a fist; (i) Temple in Thailand; (j) Restaurant; (k) Slope in a street. ${ }^{*} p<.05$. picture (g), it was not found at all. Figure 3 shows the pictures indicating the largest plastic effect (temple in Thailand, $83 \%$ ) and the smallest plastic effect (flower hedge, $56 \%$ ). Clearly, the temple in Thailand contained more pictorial depth cues than did the flower hedge.

The plastic effect was compared among the oculomotor conditions. With the use of the numbers of reflections that each observer judged to be greater in depth, we performed a one-way ANOVA and did not obtain a significant difference, $F(2,63)=1.40$. Although the plastic effect seemed to be greater in the restrictive-monocular condition (17.3) than in the other two conditions (15.5 and 15.7), this difference was not significant.

The response times obtained under the free-binocular condition were averaged across pictures for each observer. The mean across all observers was $15.3 \mathrm{~s}$, and the median was $13.8 \mathrm{~s}$, with a range of 7.4 to $33.0 \mathrm{~s}$. The response times were also averaged across observers for each picture. The mean for all pictures was $15.3 \mathrm{~s}$, and the median was $15.0 \mathrm{~s}$, with a range from 11.2 to 20.2 s. Figure 4 shows the mean
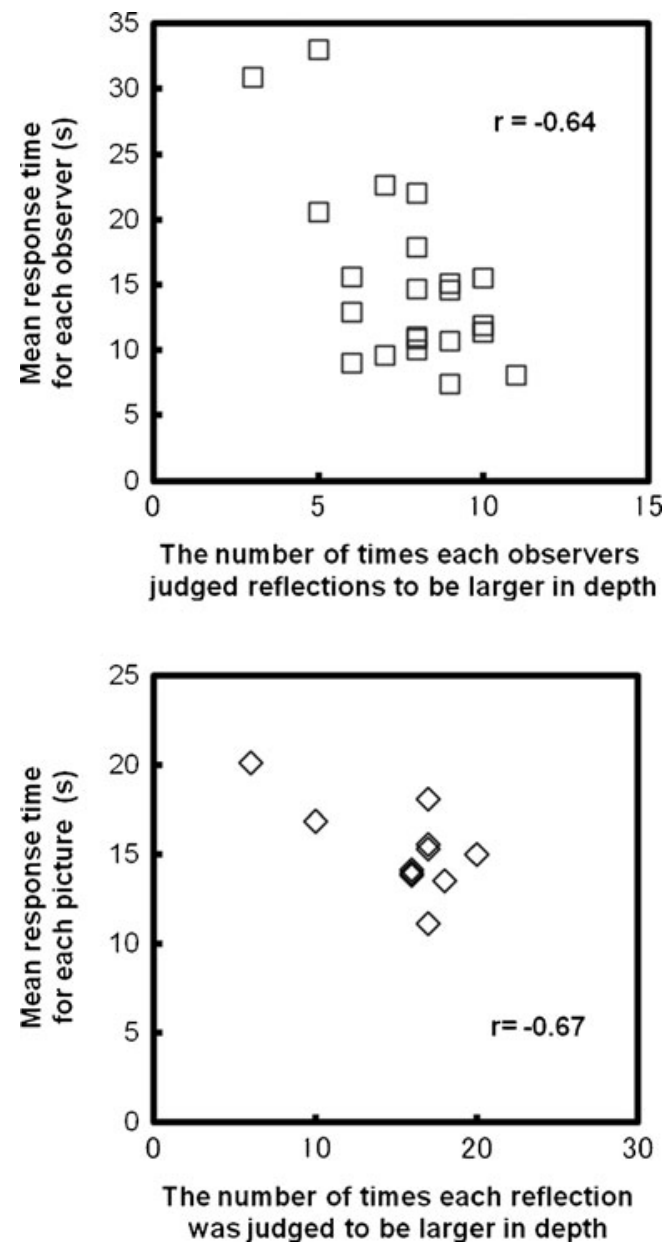

Fig. 4 Mean response times for each observer (upper panel) and for each picture (lower panel), plotted against the number of times that the reflected pictures were judged to be greater in depth 
response times for each observer (upper panel) and for each picture (lower panel) against the number of times that the reflected pictures were judged to be greater in depth - that is, the size of the plastic effect. The correlation for the upper panel $(-.64)$ was significantly different from zero, $t(20)=$ $3.73, p<.01$, and the correlation for the lower panel $(-.67)$ was also significantly different from zero, $t(9)=2.70, p<.05$.

\section{Discussion}

The plastic effect was generally confirmed: For about $73 \%$ of all judgments, the reflected pictures were perceived to be greater in depth than the directly observed pictures. In this experiment, it was assumed that all observers who had a depth set judged the reflected pictures to be greater in depth, whereas the observers who had a flatness set judged depth at chance. If the proportions of observers with a depth set and a flatness set are given as $d$ and $f$, respectively $(d+f=1)$, then the proportion of the observes who judged the reflected pictures to be greater in depth was $d+f / 2$, and the proportion of the observers who judged the directly observed pictures to be greater in depth was $f / 2$. Since $f / 2=.27$ from the present results, we obtained $f=.54$. Also, since $d+f / 2=.73$, we obtained $d=.46$.

The presence or absence of the plastic effect seemed to depend on the picture: The pictures for which the plastic effect was well detected contained ample depth cues. This result agrees with the features of perspective views that were especially painted for use of a zograscope (Oka, 1992; Screech, 1996). That is, the perspective views that have been inherited up to today were mostly drawn with very exact linear perspective.

The plastic effect did not differ among the oculomotor conditions: The detection rate of the plastic effect in the binocular condition was as high, as was that in the monocular condition. Although the plastic effect has sometimes been called "monocular stereoscopy" (Claparède, 1904; Koenderink et al., 1994) or the "monocular plastic effect" (Schlosberg, 1941), we should note that it occurs for binocular viewing as well. We also found that the detection rate of the plastic effect under restrictive monocular viewing was as high as that in the free-monocular condition. In the free-binocular and -monocular conditions, the observers were able to change the angle between the mirror and the picture, vary the viewing distance, and explore the pictures from different directions. Although these changes might make observers aware that the pictures were flat, they did not suppress the emergence of the plastic effect, but rather demonstrated its robustness.

Response times were faster for the observers who showed a larger plastic effect. Response times were also faster for the pictures whose reflections were judged to be greater in depth. This result supports the assumption that there is a negative correlation between the plastic effect and response times.
It is worth noting that the observers did not judge apparent depth immediately after the picture was presented. It took about $15 \mathrm{~s}$, on average, to judge the apparent depth of each picture. Figure 4 indicates that the mean response time was about $8 \mathrm{~s}$ for the observer who showed the largest plastic effect and was about $15 \mathrm{~s}$ for the pictures that elicited the largest plastic effect. Why were the response times so long? One possible explanation may be that the plastic effect needs time to develop fully, as would be shown in the next experiment. Another explanation may be that $56 \%$ of the observers had a flatness set and had to judge depth by guesses.

\section{Experiment 2}

In Experiment 2, we examined the time course of the plastic effect. In Experiment 1, $46 \%$ of the observers were in the depth mode of perception, whereas the others were in the flatness mode. We predicted that if the observers continued to look at a reflected picture, the number of observers who would detect the plastic effect would increase, because by prolonged exposure to the reflected picture, the observers would have a greater opportunity to focus their attention on pictorial depth cues. In Experiment 2, we used a movie as a stimulus to draw observers' attention. In addition, to compare the present results with those of the free-binocular condition of Experiment 1, the duration for which the observers were exposed to the movie approximated the total duration of exposure to the pictures in Experiment 1.

\section{Method}

Observers A group of 22 students (9 men, 13 women; mean age 20.8 years) were newly recruited as observers. They did not have any prior knowledge of the purpose of the experiment.

Apparatus and stimuli The nature documentary movie Earth, made by BBC in 2008, was presented on each of two 17-in. computer displays that lay side by side on a table. One was directly observed, and the other was observed after being reflected by two mirrors $(22 \mathrm{~cm}$ high $\times 32 \mathrm{~cm}$ wide) that were placed between the observer and the display (Fig. 5). By

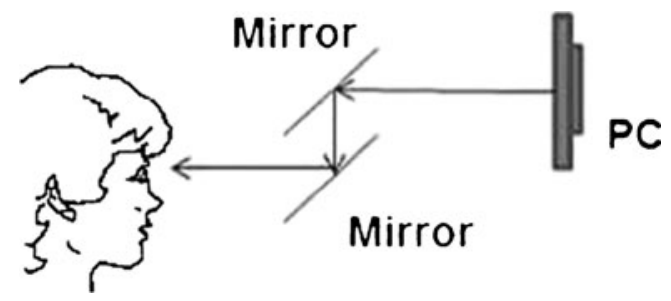

Fig. 5 Two-mirror apparatus used in Experiments 2 and 3 
the use of this mirror construction, the reflected movie had the same orientation to the eyes as the directly observed movie. The distance measured along the optical path from the observer to the display was $100 \mathrm{~cm}$ for both movies.

Procedure A newly recruited experimenter, who did not know about the expected outcome, ran the experiment. Each observer sat in front of the displays and observed them binocularly. The observer easily realized that one picture was directly presented and the other was indirectly presented after having been reflected by a mirror device. The observer first looked at the same stationary shot of the movie that was presented on each display, and was then asked to compare the shots and to report the one that was perceived to be greater (or shallower) in depth. After noting the observer's response, the experimenter started both movies simultaneously and required the observer to alternatively look at them with as equal a frequency as possible. The observer was not able to obtain simultaneous clear views of both movies, because while the observer watched one movie, the other appeared at the margin of the visual field. There was no fixation point in each display. The movies were presented for $5 \mathrm{~min}$, which approximated the total duration of exposure to the 11 pictures used in Experiment 1 [i.e., $11 \times(15 \mathrm{~s}+10 \mathrm{~s})$, where $15 \mathrm{~s}$ was the mean response time per picture and $10 \mathrm{~s}$ was the time from when the stopwatch stopped until the pictures were exchanged for the next trial]. The sound of the movies was eliminated. At 1, 2, 3, 4 , and $5 \mathrm{~min}$ after the movies had started, half of the observers reported the movie that was perceived to be greater in depth, and the remaining observers reported the movie that was perceived to be shallower in depth. For half of the observers, the directly observed display and the reflected display were placed on the left and right sides of the observers, respectively. For the remaining observers, the left-right positions of the displays were exchanged.

\section{Results and discussion}

Figure 6 shows the number of observers who judged the reflected movie or the directly observed movie to be greater in depth. The number of observers who judged the reflected movie to be greater in depth was almost at chance from the pretrial to $3 \mathrm{~min}$ into the presentation, but it increased rapidly from 4 to $5 \mathrm{~min}$, and was significantly greater than chance at 5 min (according to a binominal test, $p<.05$ ). In the early phase of this experiment, all of the observers looked at the movie in flatness mode (since $f / 2=.5$ and $d+f / 2=.5$, we obtained $f=1.0$ and $d=0.0$ ), but in the final phase, $72 \%$ of the observers (since $f / 2=$

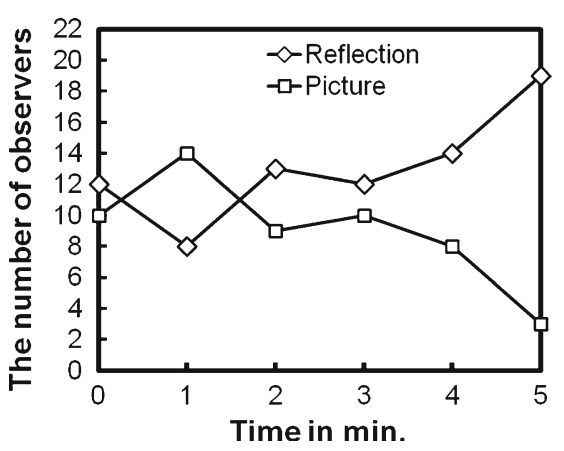

Fig. 6 Numbers of observers who judged a directly observed movie or the reflected movie to be greater in apparent depth, as a function of elapsed time in minutes

.14 and $d+f / 2=.86$, we obtained $f=.28$ and $d=.72$ ) were in depth mode.

It is possible to compare the detection rates of the plastic effect between Experiments 1 and 2. As is shown in Fig. 6, the detection rates of this experiment were $.36, .59, .55, .64$, and .86 , respectively, at $1,2,3,4$, and 5 min after the movies had started. The mean detection rate was $60 \%$. This value was somewhat smaller than the mean detection rate of binocular viewing in Experiment 1 (70\%), although the period of exposure to the movies was almost the same as that to the pictures. This difference in detection rates may be accounted for by three possible sources. The first is the number of depth judgments: Each observer of Experiment 1 made 11 judgments, whereas each observer of Experiment 2 made only five judgments. This increased repetition of judgments in Experiment 1 might have enhanced attention to the pictures and increased the opportunity to form a depth set. The second possibility is based on the finding that attention is easily captured by motion stimuli (e.g., Franconeri \& Simons, 2003; Hillstrom \& Yantis, 1994). In this experiment, the observers watched scenes in which animals, birds, and fish moved around on land, in air, and under water, respectively, and they might have been attractive to most observers. Thus, these motion scenes could have distracted the observers from attending to depth in the display. The last is a difference in depth cues contained in the pictures and the movie in this study. Most of the pictures of Experiment 1 contained persons and artificial objects like streets and houses, whereas the movie of Experiment 2 contained only natural scenes on the earth. If scenes of persons and artificial objects contain more depth cues than do natural scenes, the pictures of Experiment 1 may have been more helpful in forming a depth set.

More importantly, for the plastic effect to occur fully, $4 \mathrm{~min}$ were needed after a reflected movie had been presented to the observer. This result explains why the mean response time was as much as $15 \mathrm{~s}$ in Experiment 1: The response time was generally large on the initial trials because the plastic effect did not occur, but it gradually became smaller as the judgments 
progressed and the plastic effect began to occur. Thus, the plastic effect is not an outcome of a process that occurs immediately after stimuli are presented. The plastic effect seems to involve acquisition of a visual skill that needs a certain time to work.

\section{Experiment 3}

In Experiment 3, we examined the sustainability of the plastic effect. If the plastic effect occurs under a particular mental set, as Schlosberg (1941) suggested, it should last for a while after the observation of reflected pictures has been terminated, and it may affect the apparent depth of directly observed pictures. However, if the plastic effect is a consequence of a processing that holds only during the time of observation of the reflected pictures (i.e., is a stimulus-contingent process), it would stop when the reflected pictures are removed from the visual field. Experiment 3 dealt with this problem. All of the observers in this experiment first judged the apparent depth of directly observed pictures. Next, observers in the experimental condition were exposed to reflections of the same pictures, whereas observers in the control condition were exposed to the same real pictures. Finally, all observers judged changes in the apparent depth of directly observed pictures, relative to the remembered depth of the pictures whose depth had been judged before. If formation of a mental set is requisite for the plastic effect to occur and this set persists over time, then changes in apparent depth would be larger in the experimental than in the control condition. If the plastic effect is a product of a stimulus-contingent process, then changes in apparent depth would be the same in both conditions.

\section{Method}

Observers A group of 20 students (8 men, 12 women; mean age 21.5 years) participated in this experiment. They did not have any prior knowledge of the purpose of the experiment. None of them had participated in Experiment 1 or 2.

Apparatus and pictures A total of 15 pictures were randomly presented on a 17-in. computer display. Five newly selected pictures were added to 10 of the 11 pictures used in Experiment 1 (the downtown night scene was excluded). The added pictures contained one or two standing women against different backgrounds, including a street, an open field, a seaside, a corridor, and a destroyed building. These backgrounds seemed to differ in apparent depth. These 15 pictures were directly observed on the display or were indirectly observed after being reflected by a mirror device (see Fig. 5) that was placed between the observer and the display. The distance measured along the optical path from the observer to the display was $100 \mathrm{~cm}$.
Procedure We again recruited an experimenter who did not know the expected outcome of this experiment. She had not participated as an experimenter or observer in either of the preceding experiments.

Ten observers were randomly assigned to each of the experimental and control conditions. In the first phase, all observers followed the same procedure. Each sat in front of the display and directly observed it binocularly. The observer judged the degree of apparent depth for each picture by the use of a 5-point scale: no depth, little depth, moderate depth, great depth, and extensive depth. The observer was encouraged to judge on the basis of their whole depth impression of each picture. The observer's responses were coded with the integer numbers 0 to 4 , which corresponded to responses from no depth to extensive depth. No time limit was imposed on the observers.

In the experimental condition of the next phase, the mirror device was placed between the observer and the display. Each observer was repeatedly exposed to the 15 pictures reflected by the mirrors. These pictures were each presented 15 times in the same sequence. The observer was thus exposed to a total of $225(15 \times 15)$ reflected pictures. Each picture lasted for $1 \mathrm{~s}$ without an interstimulus interval. The total period of exposure, $3.75 \mathrm{~min}$, was decided on the grounds that it took $4 \mathrm{~min}$ for the plastic effect to occur (Exp. 2). The observers were sufficiently aware of the presence of the mirrors by which the pictures were reflected. In the control condition, each observer followed the same procedure as in the experimental condition, except for being directly exposed to the real pictures. Thus, the mirror device was not used in the control condition. In either condition, the observer was permitted to see each picture freely without a fixation point. The observer's task was simply to look at the series of the reflected or real pictures and not to make judgments to be analyzed later.

In the final phase, all observers again followed the same procedure. The same pictures were again randomly presented to each observer. Each observer directly looked at each picture and judged how its apparent depth had changed relative to the apparent depth of the pictures he or she had judged in the first phase (i.e., remembered depth). For judgments of change in depth, the observer used the 5-point scale: much reduced, reduced, no change, extended, and much extended. The observer's responses were coded with the integer numbers from -2 to +2 , which corresponded to responses from much reduced to much extended. There was no fixation point, and the observer was permitted to see each picture freely. No time limit was imposed on the observer.

\section{Results}

The ratings of apparent depth for the directly observed pictures in the initial phase were analyzed by a two-way $($ Condition $\times$ Picture) repeated measures ANOVA. The 
mean apparent depth in the experimental condition, 2.09 ( $S D=0.86$, range 0.3 to 3.3 ), was not significantly different from the mean apparent depth in the control condition, 2.04 $(S D=0.80$, range 0.2 to 3.2$)$. The main effect of picture was significant, $F(14,252)=14.16, p<.001$, suggesting that the apparent depth varied from picture to picture.

The changes in apparent depth for the directly observed pictures that were judged in the final phase were analyzed in a two-way (Condition $\times$ Picture) repeated measures ANOVA. The mean change in apparent depth obtained after exposure to the reflected pictures, $0.49(S D=0.33)$, was significantly different from the mean change in apparent depth obtained after exposure to the real pictures, 0.20 $(S D=0.29), F(1,18)=8.05, p<.05$. This means that the plastic effect was transferred to normal, flat pictures. The main effect of picture was significant, $F(14,252)=1.86$, $p<.05$, suggesting that the mean change in apparent depth varied from picture to picture. The Condition $\times$ Picture interaction was not significant, $F(14,252)=1.10$.

Figure 7 shows the relation between the mean apparent depth of the directly observed pictures in the first phase and the mean change in apparent depth of the directly observed pictures in the last phase. The upper panel is for the experimental condition, and the lower panel is the control
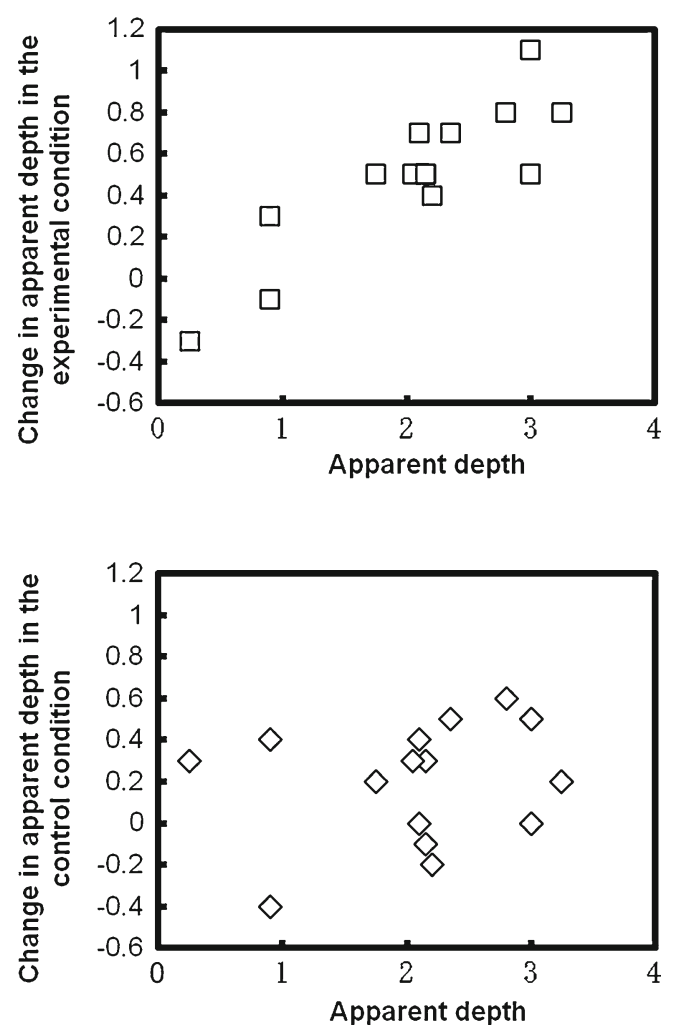

Fig. 7 Changes in apparent depth of directly observed pictures, as judged after prolonged exposure to the reflected (upper panel) or to the real (lower panel) pictures, plotted against the apparent depth of the pictures condition. The data points represent the pictures in either panel. The correlation for the experimental condition was .88 , which was significantly different from zero, $t(13)=6.68, p<.001$, whereas the correlation for the control condition was .20 , which was not significantly different from zero. These results suggest that the transfer of the plastic effect that had occurred while looking at the reflected pictures was emphasized for pictures that contained many depth cues.

\section{General discussion}

The results of Experiment 1 indicated that the plastic effect was equally well detected across free binocular, free monocular, and restrictive monocular viewing. Overall, it was detected on about $73 \%$ of the trials. Although Kaldenbach (1985) speculated that a mirror does not play an essential role in a zograscope, this is utterly incorrect, as was clearly demonstrated by the present study. Binocular viewing provided more flatness cues than did monocular viewing, but the detection rate of the plastic effect did not reduce in binocular viewing. This result cannot be accounted for by a reduction of flatness cues. A possible explanation is that the apparent absolute distance of reflected picture was unsettled by the double locations of the reflected pictures, and the apparent depth was increased by pictorial depth cues in any oculomotor condition. We think that Experiment 1 was successful in isolating the effects of a mirror in the plastic effect.

Despite the results of Experiment 1, we hesitate to deny a direct effect of oculomotor cues, because there is evidence that the plastic effect can be accounted for by oculomotor cues (Ames, 1925; Claparède, 1904; Koenderink et al., 1994). Under the present circumstance, it may be safe to assume that the plastic effect is compounded of the effect of a mirror and the effect of oculomotor cues. Each of these effects can be investigated by means of a specific comparison. To examine how a mirror contributes to the plastic effect, as in the present study, apparent depth should be compared between a real picture and the reflected picture. To examine how oculomotor cues contribute to the plastic effect, the apparent depth of a real or reflected picture should be compared between monocular and binocular viewings. From this point of view, it is possible to understand the results of the Koenderink et al. (1994) study. The difference between binocular viewing through a synopter and natural binocular viewing may partially be attributed to the effect of mirrors, whereas the difference between natural monocular and binocular viewings may be due to the effects of oculomotor cues.

The results of Experiments 2 and 3 indicated two temporal properties of the plastic effect. First, the effect tended not 
to occur at the moment a reflected picture was presented; 4 min were required for the plastic effect to develop fully. Second, the plastic effect that had been formed by observing reflected pictures transferred to directly observed pictures and persisted after the reflected pictures had been removed. The amount of transfer was larger for pictures that represented greater apparent depth $(r=.88)$. It seems that the plastic effect involves a learning process that needs time for acquisition and that can transfer to real pictures.

One interesting finding is seemingly opposite to the plastic effect but is explainable in terms of the double locations of a reflected picture: When a reflected target is seen in a natural environment, it appears closer to the observer than does the directly observed target (Higashiyama \& Shimono, 2004; Higashiyama, Yokoyama, \& Shimono, 2001). In the mentioned studies, as is shown in Fig. 8, the distance of the comparison, which was directly observed, was matched to the distance of a standard, which was reflected by a mirror. The comparison was then placed somewhere between the actual position of the mirror and the virtual image of the standard. We call this the "foreshortening effect of absolute distance in three-dimensional scenes." To explain this finding, we assume that the standard is doubly localized both where the mirror is placed and where the virtual image is formed, and a perceptual compromise then occurs between the locations.

The plastic effect may have several practical applications. Hagen, Jones, and Reed (1978), Takezawa (2005), and Watanabe $(2004,2006)$ indicated that apparent depth in a picture is compressed as compared to apparent depth in ordinary environments (cf. Hecht, van Doorn, \& Koenderink, 1999). To compensate for this compression in apparent depth, a mirror may be useful. In Experiment 2, a number of our observers spontaneously reported, with surprise, that the reflected movie was more plastic than the directly watched movie. Their reports agree with what Ponzo (1911) experienced in a movie theater in which the movie on the screen happened to be reflected by a mirror at the side of the screen. The theater was probably so dark that he could not see the mirror frame, and the mirror was so far away that its absolute location was uncertain. These theater conditions, in addition to the effects of the mirror, might reduce flatness cues (Eby \& Braunstein, 1995; Hagen et al., 1978; Lumsden, 1983) and help enhance the plastic effect.

As mentioned in the introduction, the plastic effect has often been observed with various optic devices. In some of these devices, not only a mirror but also a convex lens has been incorporated, as can be seen in Fig. 1, but we have little knowledge on the role of a convex lens in the plastic effect. Informal observation, however, has given us an impression that a convex lens may also enhance the plastic effect. When a picture is seen through a convex lens, its virtual image is mislocalized and the state of accommodation of the eye may be changed, and the image is also skewed at the margin of the visual field. Thus, what should be done in a future study is to find out how these variables generated by the convex lens contribute to the plastic effect.

To sum up, we have elucidated the nature of the plastic effect, which has rarely been explored scientifically. In particular, we obtained two findings regarding the role of a mirror. First, the plastic effect developed well for pictures reflected by a single mirror and was equally detected across oculomotor conditions. Additionally, the effect was detected not only for static pictures, but also for pictures in motion, as in a movie. We interpreted this finding as suggesting that the doubling of the locations of a picture caused by a mirror makes the absolute distance of the picture unsettled, activates depth cues in the picture, and suppresses flatness cues. Second, 4 min were required for the plastic effect to develop fully, and once the effect had occurred, it transferred to directly observed pictures. We also interpreted this finding as suggesting that the plastic effect involves not a simple stimulus-contingent process, but rather a perceptuallearning process regarding a way of perceiving.
Fig. 8 Foreshortening effect of absolute distance in a threedimensional environment.

When the distance of a comparison, which was seen directly, was matched to the distance of a standard $(20 \mathrm{~m})$, which was reflected by a mirror, the comparison tended to be placed at a distance of $12 \mathrm{~m}$, which was between the mirror and the virtual image of the standard (Higashiyama et al., 2001)

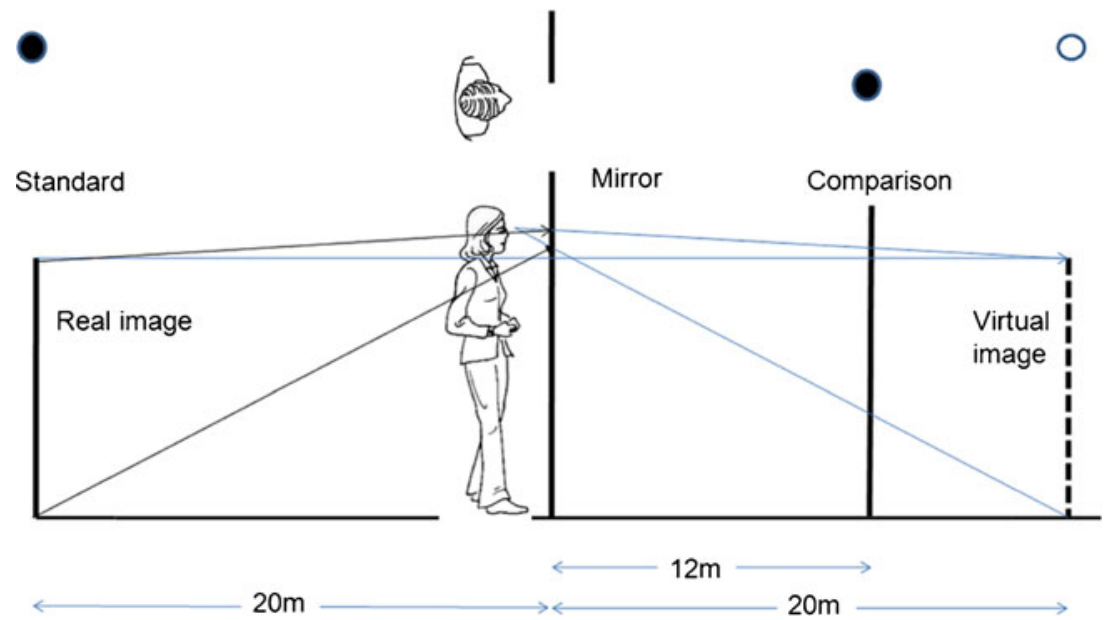


Author note The mirror device used in Experiments 2 and 3 was manually made by Takáaki Ito and was kindly provided for this study. We also appreciate Heiko Hecht, who kindly sent us the Zoth article and made comments on an earlier version of our manuscript. This study was supported by Grant-in-Aid for Scientific Research No. 23530965 from the Japan Society for the Promotion of Science.

\section{References}

Allison, R. S., Gillam, B. J., \& Vecellio, E. (2009). Binocular depth discrimination and estimation beyond interaction space. Journal of Vision, 9(1), 10:1-14. doi:10.1167/9.1.10

Ames, A., Jr. (1925). The illusion of depth from single pictures. Journal of the Optical Society of America, 10, 137-148. doi:10.1364/JOSA.10.000137

Bülthoff, H. H., \& Mallot, H. A. (1988). Integration of depth modules: Stereo and shading. Journal of the Optical Society of America. A, 5, 1749-1758. doi:10.1364/JOSAA.5.001749

Carl Zeiss Jena (1907). Instrument zum beidäugigen Betrachten von Gemälden u.ggl. Kaiserliches Patentamt, Patentschrift Nr 194480, Klasse 42h, Gruppe 34.

Chaldecott, J. A. (1953). The zograscope or optical diagonal machine. Annals of Science, 9, 315-322. doi:10.1080/00033795300200243

Claparède, E. (1904). Stéréoscopie monoculaire paradoxale. Annales d'Oculistique, 132, 465-466.

Clayton, T. (1997). The English print 1688-1802. New Haven: Yale University Press.

Eaton, E. M. (1919). The visual perception of solid form. British Journal of Ophthalmology, 3, 349-363 and 399-408.

Eby, D. W., \& Braunstein, M. L. (1995). Perceptual flattening of threedimensional scenes enclosed by a frame. Perception, 6, 657-660.

Emerson, E. (1863). On the perception of relief. British Journal of Photography, 10, 10-11.

Foley, J. M. (1980). Binocular distance perception. Psychological Review, 87, 411-434. doi:10.1037/0033-295X.87.5.411

Franconeri, S. L., \& Simons, D. J. (2003). Moving and looming stimuli capture attention. Perception \& Psychophysics, 65, 999-1010. doi:10.3758/BF03194829

Frisby, J. P., Buckley, D., \& Duke, P. A. (1996). Evidence for good recovery of lengths of real objects seen with natural stereo viewing. Perception, 25, 129-154.

Gibson, J. J. (1979). The ecological approach to visual perception (chap. 15). Boston: Houghton Mifflin.

Gogel, W. C. (1962). Convergence as a determiner of perceived absolute size. Journal of Psychology, 53, 91-104.

Grant, V. W. (1942). Accommodation and convergence in visual space perception. Journal of Experimental Psychology, 31, 89-104. doi: $10.1037 / \mathrm{h} 0062359$

Haber, R. N. (1980). How we perceive depth from flat pictures. American Scientist, 68, 370-380.

Hagen, M. A., Jones, R. K., \& Reed, E. S. (1978). On the neglected variable in theories of pictorial perception: Truncation of the visual field. Perception \& Psychophysics, 23, 326-330. doi:10.3758/BF03199716

Hecht, H., van Doorn, A. J., \& Koenderink, J. J. (1999). Compression of visual space in natural scenes and in their photographic counterparts. Perception \& Psychophysics, 61, 1269-1286. doi:10.3758/BF03206179

Higashiyama, A. (1984). The effects of familiar size on judgments of size and distance: An interaction of viewing attitude with spatial cues. Perception \& Psychophysics, 35, 305-312. doi:10.3758/ BF03206333
Higashiyama, A., \& Shimono, K. (2004). Mirror vision: Perceived size and perceived distance of virtual images. Perception \& Psychophysics, 66, 679-691. doi:10.3758/BF03194911

Higashiyama, A., Yokoyama, Y., \& Shimono, K. (2001). Perceived distance of targets in convex mirrors. Japanese Psychological Research, 43, 13-24. doi:10.1111/1468-5884.00155

Hillstrom, A. P., \& Yantis, S. (1994). Visual motion and attentional capture. Perception \& Psychophysics, 55, 399-411. doi:10.3758/ BF03205298

Kaldenbach, C. J. (1985). Perspective views. Print Quarterly, 2, 87-104.

Koenderink, J. J., van Doorn, A. J., \& Kappers, A. M. L. (1992). Surface perception in pictures. Perception \& Psychophysics, 52, 487-496. doi:10.3758/BF03206710

Koenderink, J. J., van Doorn, A. J., \& Kappers, A. M. L. (1994). On so-called paradoxical monocular stereoscopy. Perception, 23, 583-594. doi:10.1068/p230583

Koenderink, J. J., van Doorn, A. J., \& Kappers, A. M. L. (1995). Depth relief. Perception, 24, 115-126. doi:10.1068/p240115

Komoda, M. K., \& Ono, H. (1974). Oculomotor adjustments and sizedistance perception. Perception \& Psychophysics, 15, 353-360. doi:10.3758/BF03213958

Lumsden, E. A. (1983). Perception of radial distance as a function of magnification and truncation of depicted spatial layout. Perception \& Psychophysics, 33, 177-182. doi:10.3758/BF03202836

McKee, s. P., \& Taylor, D. G. (2010). The perception of binocular and monocular depth judgments in natural settings. Journal of Vision, 10(10), 5:1-13. doi:10.1167/10.10.5

Niederée, R., \& Heyer, D. (2003). The dual nature of picture perception: A challenge to current general accounts of visual perception. In H. Hecht, R. Schwartz, \& M. Atherton (Eds.), Looking into pictures: An interdisciplinary approach to pictorial space (pp. 77-98). Cambridge: MIT Press.

Oka, Y. (1992). A new approach to optical prints [Megane-e shinko: Ukiyoeshi tachi ga nozoita seiyo]. Tokyo: Chikuma Shobo. (in Japanese)

Pirenne, M. H. (1970). Optics, paintings and photography (chap. 8). Cambridge: Cambridge University Press.

Ponzo, M. (1911). Un appareil pour la vision plastique de photographies. Archives Italiennes de Biologie, 56, 125-126.

Rock, I. (1984). Perception (chap. 4). New York: Scientific American Books.

Schlosberg, H. (1941). Stereoscopic depth from single pictures. The American Journal of Psychology, 54, 601-605.

Screech, T. (1996). The Western scientific gaze and popular imagery in later Edo Japan: The lens within the heart. Cambridge: Cambridge University Press.

Stevens, K. A., \& Brooks, A. (1987). Probing depth in monocular images. Biological Cybernetics, 56, 355-366. doi:10.1007/BF00319515

Swenson, H. A. (1932). The relative influence of accommodation and convergence in the judgment of distance. The Journal of General Psychology, 7, 360-380. doi:10.1080/00221309.1932.9918473

Takezawa, T. (2005). Perception of the distance of human figures in photographs [Seishi gazo-jo no jinbutsu ni taisuru okuyuki chikaku no kyori]. Japanese Journal of Psychonomic Science, 23, 177-182 (in Japanese with English abstract).

Tscherning, M. (1904). Physiologic optics (p. 321). Philadelphia: The Keystone.

von Kalpinska, L. (1910). Experimentelle Beiträge zur Analyse der Tiefenwahrnehmung. Zeitschrift für Psychologie, 57, 1-88.

Watanabe, T. (2004). Anisotropy in depth perception of photograph [Shashin no okuyuki chikaku ni okeru kukan no ihosei ni tsuite]. Japanese Journal of Psychology, 75, 24-32 (in Japanese with English abstract).

Watanabe, T. (2006). Geometrical structures of photographic and stereoscopic spaces. Spanish Journal of Psychology, 9, 263-272.

Zoth, O. (1916). Ein einfaches "Plastoskop". Zeitschrift für Sinnesphysiologie, 49, 85-88. 